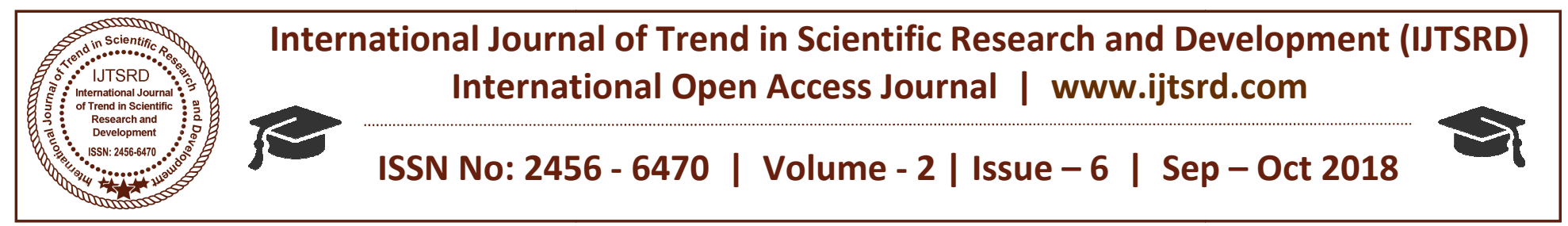

\title{
Artificial Intelligence: Basics and Terminology
}

\author{
Durgesh Raghuvanshi
}

Btech, Department of computer science,

IILM Academy of Higher Learning, Greater Noida, Uttar Pradesh, India

\section{ABSTRACT}

The Artificial intelligence field continues to be plagued by what can only be described as 'bold promises for the future syndrome'. Research in AI has built upon the tools and techniques of many different disciplines, including formal logic, probability theory, decision theory, management science, linguistic philosophy.

However, the application of these disciplines in artificial intelligence has necessitated the development of many enhancements and extensions. Since the invention of computers or machines, their capability to perform various tasks went on growing exponentially. Humans have developed the power of computer systems in terms of their diverse working domains, their increasing speed and reducing size with respect to time.

\section{INTRODUCTION}

A branch of computer science named Artificial intelligence John McCarthy, it is "The science and engineering of making intelligent machines, especially intelligent computer programs". AI is a way of making a computer, a computer-controlled robot, or a software think intelligently, in the similar manner intelligent humans think. AI is an accomplished by studying how human brain thinks, and how humans learn, decide and work while trying to solve a problem and then using the outcomes of this study as a basis of developing intelligent software and systems.

To lay claim to have developed an intelligent system is to open a hornet's nest of debate which has raged since the early days of AI. This is not surprising since one can very quickly stray into the realms of philosophy, metaphysics and or religion we do not propose to add to the debate here but to simply make ours by way of practical methods.

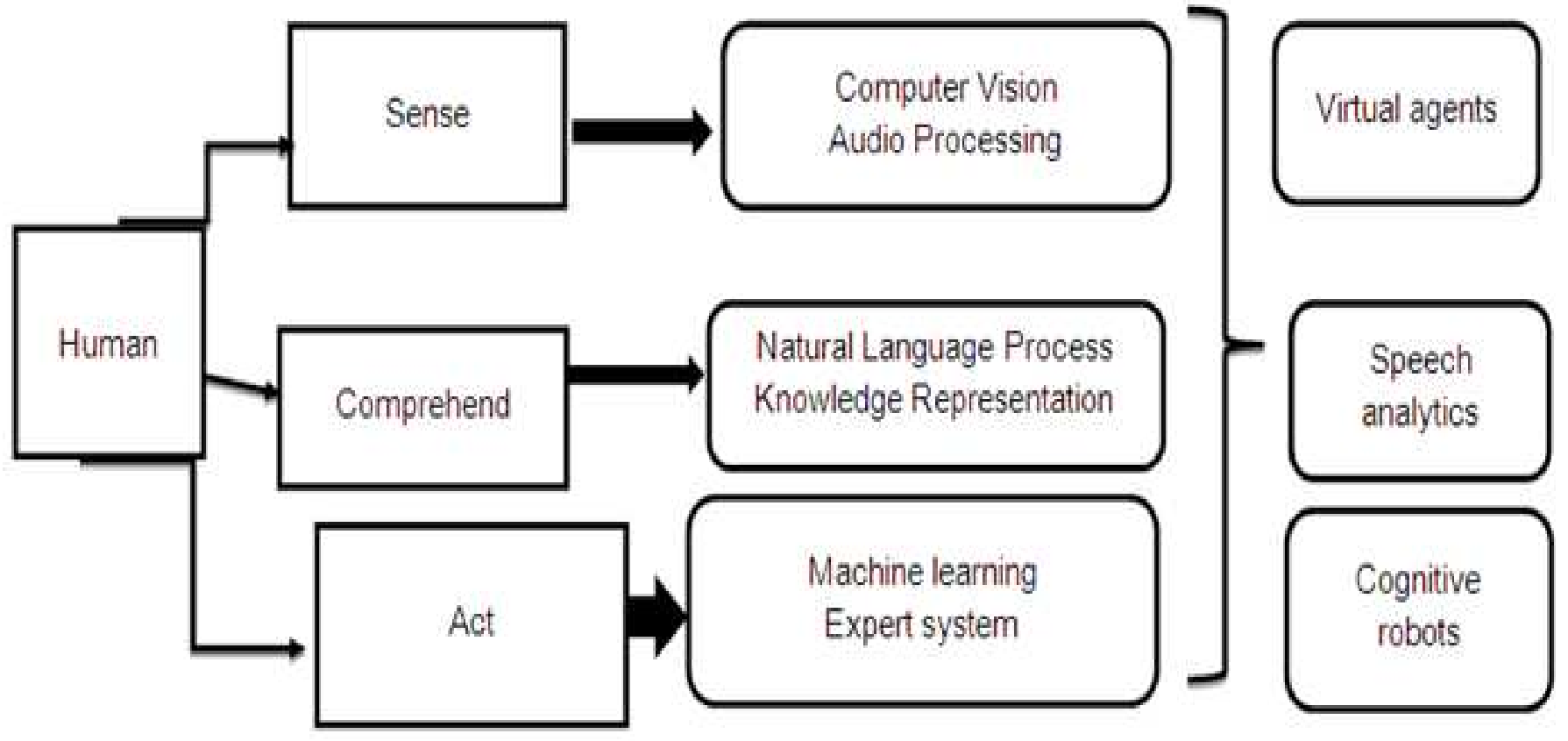


AI programming languages include LISP developed in the 1950 'S is the early programming language strongly associated with AI. LISP is a functional programming language with procedural extensions. LISP was specially designed for processing heterogeneous lists typically a list of symbols. Features of LISP are run-time type checking. AI is a new electronic machine that stores a large amount of information and processes it at very high speed. AI has the ability to solve problems hence it is the science and engineering of making intelligent machines, especially intelligent computer programs. It is related to the similar task of using computers to understand human intelligence.

\section{Types of artificial intelligence:-}

AI can be classified in any number of ways there are two types of classification.

\section{TYPE 1:-}

\section{WEAK AI OR NARROW AI:-}

$>$ It is focused on one narrow task, the phenomenon that machines which are not too intelligent to do their own work can be built in such a way that they seem smart.

$>$ An example would be a poker game where a machine beats human where in which all rules and moves are fed into the machine. There each and every possible scenario need to be entered beforehand manually. Each and every weak AI will contribute to the building of strong AI.

\section{STRONG AI:-}

The machines that can actually think and perform tasks on its own just like a human being. There are no proper existing examples ofthis, but some industry leaders are very keen on getting close to build a strong AI which has resulted in rapid progress.

\section{TYPE 2 (BASED ON FUNCTIONALITIES) :-}

\section{$>$ Reactive machines:-}

$>$ This is one of the basic forms of AI. It doesn't have post-memory and cannot use past information to perform future actions.

$>$ Example: - IBM chess program that beat Garry Kasparov in the 1990's.

\section{$>$ Limited Memory:-}

$>$ AI systems can use past experiences to inform future decisions. Some of the decision-making functions in self-driving cars have been designed this way. Observations used to inform actions happening in the not so distant future, such as a car that has changed lanes. These observations are not stored permanently and also apple chatbot Siri.

\section{Theory of Mind:-}

$>$ This type of AI should be able to understand people's emotion, belief thoughts, expectations and be able to interact socially. Even though a lot of improvements are there in this field this kind of AI is not completed yet.

\section{Self-awareness:-}

$>$ An AI that has its own conscious super intelligent, self-awareness sentiment. Of course, this kind of bot also doesn't exist and if achieved it will be one of the milestones in the field of AI.

\section{IMPORTANCE OF AI:-}

\section{Game playing:-}

- You can buy machines that can play a master level of chess for a few hundred dollars. There is some $\mathrm{Ai}$ in them but they ay well against people mainly through brute force computation looking at hundreds of thousands of positions. To beat a world champion by brute force and known reliable heuristics requires being able to look at 200 million positions per second.

\section{Speech recognition:-}

In the 1990's computer speech recognition reached a practical level for limited purposes. The United -Airlines has replaced its keyboard tree for flight information by a system using speech recognition of flight numbers and city names.

\section{Understanding Natural Language:-}

Just getting a sequence of words into a computer is not enough. Parsing sentences is not enough either the computer has to be provided with an understanding of the domain the text is about and this is presently possible only for very limited domains.

\section{$>$ Computer Vision:-}

$>$ The world is composed of $3 \mathrm{~d}$ objects but the inputs to the human eye and computers TV cameras are 2D some useful programs can work solely in two dimensions but full computer vision requires partial $3 \mathrm{~d}$ information that is not just a set of $2 \mathrm{~d}$ views. At present, there are only limited ways of representing $3 \mathrm{~d}$ information directly and they are not as good as what humans evidently use. 


\section{WHAT CONTRIBUTES AI?}

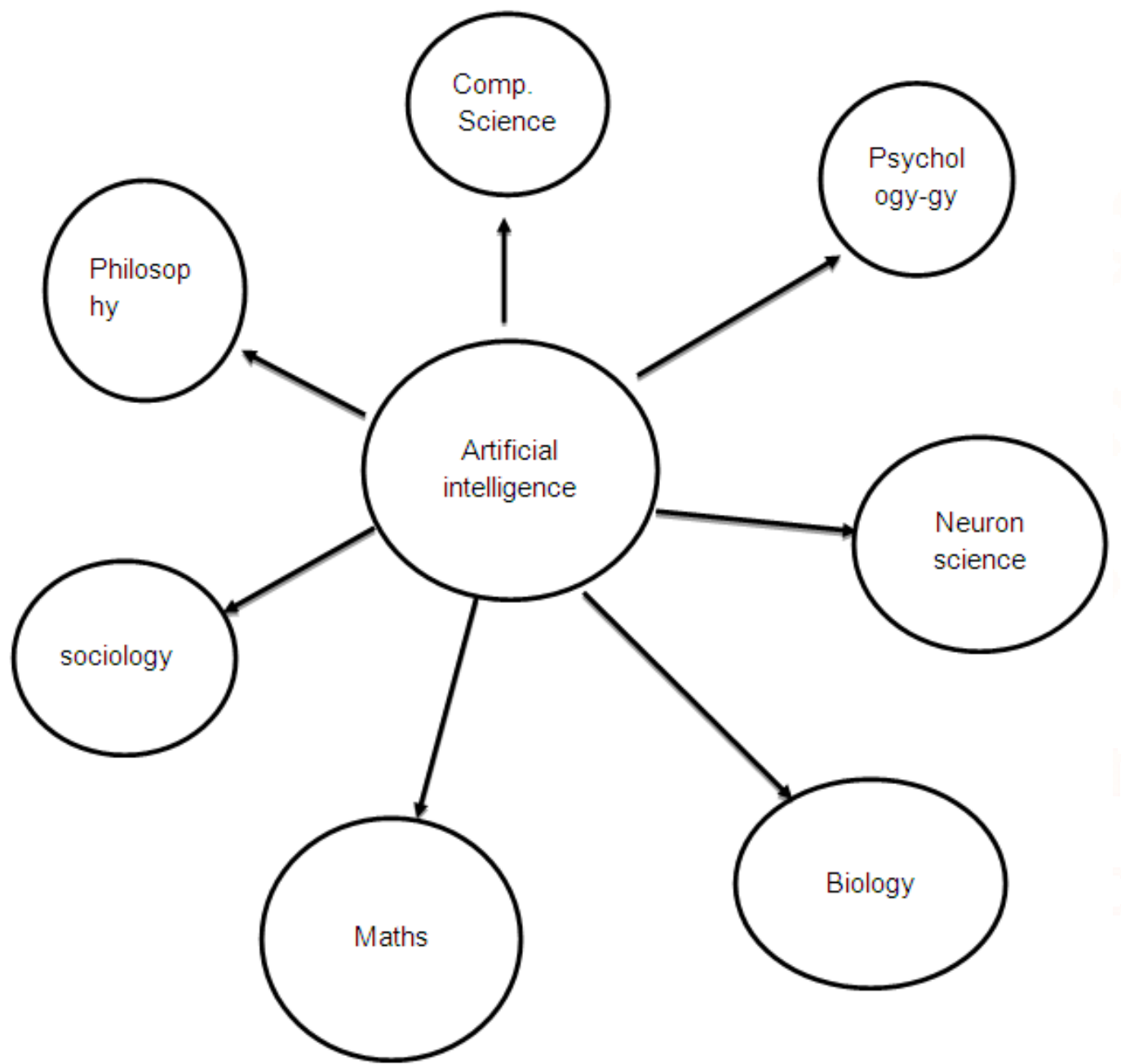

Artificial intelligence is a science and technology based on disciplines such as Computer science, biology, psychology, linguistics, mathematics, and engineering. A major thrust of artificial intelligence is in the development of computer functions associated with human intelligence such as reasoning, learning problem-solving. Out of the following areas, one or multiple areas can contribute to building an intelligent system.

\section{Conclusion:-}

I have sketched two ways in which the ALP agent model, building upon many different developments in AI, can be used by many ordinary people to improve their own human intelligence It can help them express their thoughts more clearly and coherently and it can help them to make better choices. I believe that the application of such techniques as a fruitful area of collaboration between researchers in AI and researchers in more humanistic disciplines.

\section{Reference:-}

1. Robert Kowalski, Oxford University wellington square united kingdom

2. Analilia Lauren cruces, the researcher in computer science, University of Wollongong Australia.

3. John Fulcher, intelligent systems research center university of Wollongong Australia.

4. Professor Pradipta Das, Veer Surendrasai University of technology, Sambalpur Odisha India. 